\title{
Application of a New Wavelet Threshold Method in Unconventional Oil and Gas Reservoir Seismic Data Denoising
}

\author{
Guxi Wang, ${ }^{1,2}$ Ling Chen, ${ }^{1}$ Si Guo, ${ }^{1,3}$ Yu Peng, ${ }^{3}$ and Ke Guo ${ }^{1}$ \\ ${ }^{1}$ Chengdu University of Technology, Chengdu 610059, China \\ ${ }^{2}$ Nation Institute of Measurement and Testing Technology, Chengdu 610021, China \\ ${ }^{3}$ CNPC Chuanqing Drilling Engineering Company Limited, Chengdu 610000, China
}

Correspondence should be addressed to Ling Chen; chenl@cdut.edu.cn

Received 5 January 2015; Accepted 27 February 2015

Academic Editor: Yun-Bo Zhao

Copyright (C) 2015 Guxi Wang et al. This is an open access article distributed under the Creative Commons Attribution License, which permits unrestricted use, distribution, and reproduction in any medium, provided the original work is properly cited.

\begin{abstract}
Seismic data processing is an important aspect to improve the signal to noise ratio. The main work of this paper is to combine the characteristics of seismic data, using wavelet transform method, to eliminate and control such random noise, aiming to improve the signal to noise ratio and the technical methods used in large data systems, so that there can be better promotion and application. In recent years, prestack data denoising of all-digital three-dimensional seismic data is the key to data processing. Contrapose the characteristics of all-digital three-dimensional seismic data, and, on the basis of previous studies, a new threshold function is proposed. Comparing between conventional hard threshold and soft threshold, this function not only is easy to compute, but also has excellent mathematical properties and a clear physical meaning. The simulation results proved that this method can well remove the random noise. Using this threshold function in actual seismic processing of unconventional lithologic gas reservoir with low porosity, low permeability, low abundance, and strong heterogeneity, the results show that the denoising method can availably improve seismic processing effects and enhance the signal to noise ratio (SNR).
\end{abstract}

\section{Introduction}

The study area is located in the middle of Sulige gas field, with small effective reservoir thickness, expressed as "thin, more scattered, and miscellaneous" feature on the vertical of reservoir; therefore, to remove noise in seismic data, which improves the reliability of the signal, is particularly important for reservoir prediction in later work, as it is always the goal of geophysical workers.

Random noise cannot seek a unified time pattern, with wide bandwidth, and cannot be determined by its apparent velocity and direction of propagation, thus removing random noise and the key point is to improve its SNR. In order to effectively remove random noise, various denoising methods have been proposed; thereinto, the wavelet transform method works better in recent years. Wavelet transform theory developed in mid and late eighties of last century [1], and it can make multiscale decomposition of seismic signals.

Since desired signal and random noise have different characteristics on different wavelet scales, such as during valid signal occurs within a short time, instantaneous modulus maxima of the wavelet coefficients occurs, and with the decomposition scale increment, wavelet coefficients and dense maxima of random noise in desired signal will decrease while decomposition scale increasing $[2,3]$. Wavelet denoising method separates desired signal and random noise by this opposite characteristic between them. In 1995, Donoho proposed wavelet threshold method, and soon it became one of the most widely used methods for it is easy to implement and with fast calculation speed.

But hard and soft threshold methods commonly used in wavelet threshold methods have some shortcomings, such as the following: remodeling factor in hard threshold processing occurs intermittently at the threshold point; the reconstructed signal is prone to Pseudo-Gibbo phenomenon; while although reconstruction coefficient of soft threshold has good overall continuity, there is always a constant bias between reconstructed coefficients and decomposition coefficients [4]. These all impact the wavelet threshold effect. This paper gives a new threshold function, which not only is easy 


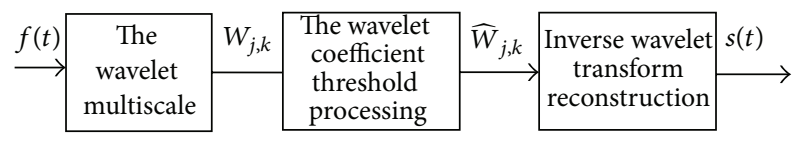

FIGURE 1: The flow of wavelet threshold.

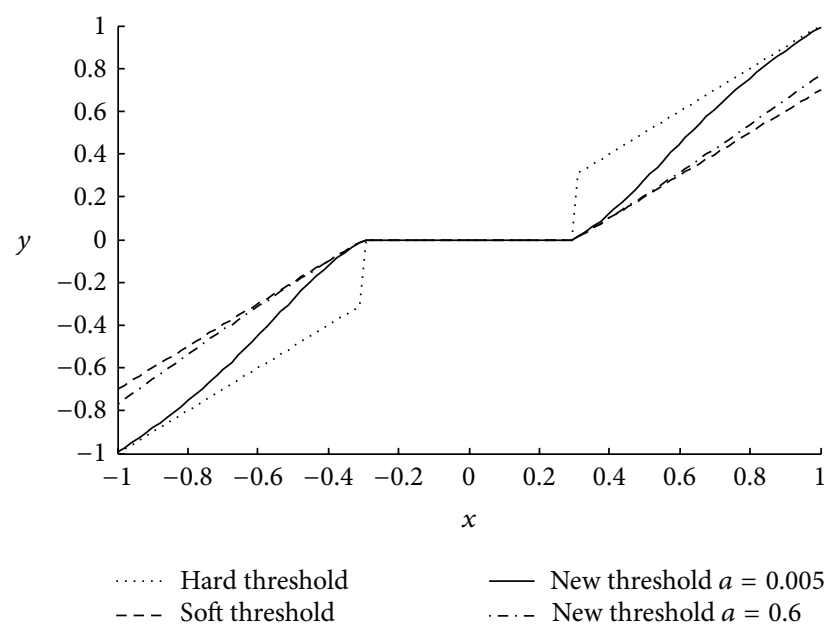

Figure 2: The new threshold.

to compute, but also has excellent mathematical properties and a clear physical meaning. The significant effect of the new threshold function will show in simulation results and practical applications.

\section{An Improved Method of Threshold Function}

Consider the following observation signal:

$$
f(t)=s(t)+n(t) .
$$

$f(t)$ is noisy random signal. $s(t)$ is original signal. $n(t)$ obeys Gaussian white noise distribution, means $N\left(0, \sigma^{2}\right)$ distribution. For one-dimensional random signal $f(t)$, we first discretize samples to give $N$ points of discrete signal $f(n), n=0,1,2, \ldots, N-1$. Then we have a wavelet transform:

$$
W f(j, k)=2^{-j / 2} \sum_{n=0}^{N-1} f(n) \psi\left(2^{-j} n-k\right)
$$

where $W f(j, k)$ are wavelet coefficients, abbreviated as $W_{j, k}$ to simplify the calculation, Making discrete wavelet transform for noisy random signals as $f(t)=s(t)+n(t)$, for wavelet transform is a linear transformation, the obtained $W_{j, k}$ are still composed by two parts: wavelet coefficients corresponding to desired signal $s(t)$, denoted by $U_{j, k}$, and wavelet coefficients corresponding to noise $n(t)$, denoted by $V_{j, k}$.

Wavelet threshold method based on the minimum mean square error (MSE) proposed by Donoho has been widely used. The basic flow is shown in Figure 1 [5].
Hard threshold function is

$$
\widehat{W}_{j, k}= \begin{cases}W_{j, k}, & \left|W_{j, k}\right| \geq k, \\ 0, & \left|W_{j, k}\right|<k\end{cases}
$$

Soft threshold function is

$$
\widehat{W}_{j, k}= \begin{cases}\operatorname{sgn}\left(W_{j, k}\right) \cdot\left(\left|W_{j, k}\right|-\lambda\right), & \left|W_{j, k}\right| \geq \lambda \\ 0, & \left|W_{j, k}\right|<\lambda\end{cases}
$$

The value $\lambda$ of formulas (3) and (4) is the preset threshold. $\operatorname{Sgn}(*)$ in formula (4) means the sign function.

A weighted average threshold estimation method is given in literatures $[6,7]$; that is,

$$
\begin{aligned}
\widehat{W}_{j, k} & \begin{cases}(1-\mu) W_{j, k}+\mu \operatorname{sgn}\left(W_{j, k}\right)\left(\left|W_{j, k}\right|-\lambda\right), & \left|W_{j, k}\right| \geq \lambda, \\
0, & \left|W_{j, k}\right|<\lambda .\end{cases}
\end{aligned}
$$

Traditional weighted average methods generally set the weighting factor $\mu$ to 0.5 , and the constant $\mu$ can overcome soft and hard threshold disadvantages in a certain extent, but it still has flaw: first threshold function is still not a continuous function, followed by a fixed bias which is still existent between estimate value and original value of wavelet coefficients, but the deviation is less than 0.5. In order to better show advantages of weighted average threshold estimation method, this paper presents a new weighting factor calculation formula:

$$
\mu=\alpha^{\left(\left|W_{j, k}\right|-\lambda\right)^{2}}, \quad(0 \leq \alpha \leq 1), 0 \leq \mu \leq 1 .
$$

The new threshold function has the following characteristics:

(1) When $\alpha \rightarrow 0$ is the hard threshold function, $\alpha \rightarrow$ 1 is the soft threshold function.

(2) $\left|\widehat{W}_{j, k}\right|$ will be gradually increased as $\left|W_{j, k}\right|$ is increasing; when $\left|W_{j, k}\right| \rightarrow \infty$, then $\widehat{W}_{j, k} \rightarrow W_{j, k}$.

(3) When $\left|W_{j, k}\right|=\lambda, \widehat{W}_{j, k}=0$; when $\left|W_{j, k}\right| \rightarrow \lambda, \widehat{W}_{j, k} \rightarrow$ 0 ; so $\widehat{W}_{j, k}$ is consecutive when $\left|W_{j, k}\right|=\lambda$.

This shows that the new threshold function is an adjustable function between hard and soft threshold function (Figure 2). As long as there is a proper sizing $\alpha$, we can get a better denoising effect, and it also has a very clear physical meaning.

\section{Simulation and Comparison}

To verify effect of the new threshold denoising function, we use Matlab toolbox containing Gaussian noise Heavisine signals. Using traditional hard and soft threshold function and the new threshold function make denoising tests. Then comparative analysis of results, select sym6 to make wavelet 
TABLE 1: Methods of signal to noise ratio (SNR/dB) and mean square error (MSE).

\begin{tabular}{lcccc}
\hline Threshold & Plus noise signal & Hard threshold & Soft threshold function & The new threshold \\
\hline SNR & 14.8606 & 24.7590 & 25.2749 & 26.5035 \\
RMSE & 1.0324 & 0.3303 & 0.3113 & 0.2702 \\
\hline
\end{tabular}
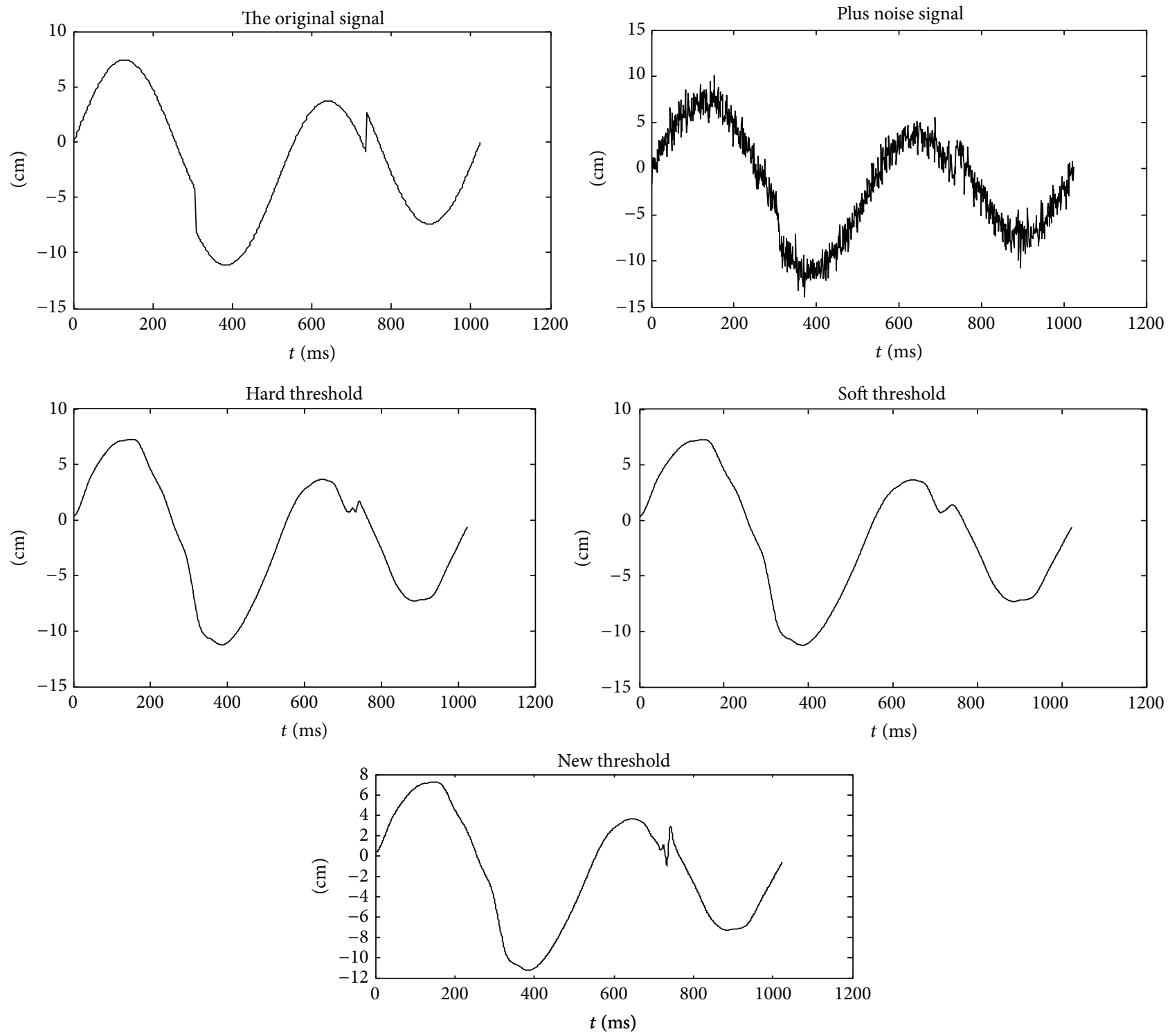

FIgURE 3: The effect diagram of different threshold function.

decomposition, the maximum resolution scale $J$ is taken as 5 , the selected threshold value of $\lambda=\sigma \sqrt{2 \log N} / \log (j+$ 1 ), wherein the noise variance $\sigma$, and $N$ is a discrete signal sampling length. The simulation results shown in Figure 3, SNR, and MSE of denoising signal are shown in Table 1.

By the numerical denoising effect evaluation in 3-1 one can see several improved threshold function denoising effects showing soft, hard threshold method is better than the classical one note. We note that the denoising effects of exponential threshold function are better than other kinds of methods; and the method of the new threshold function in root mean square error slightly inferior to the exponential threshold function, but in the signal to noise ratio of this index, is obviously better than the exponential threshold method [5].

\section{Noising Seismic Data and Application}

\subsection{Original Data Analysis}

4.1.1. Shot Record Quality Analysis. Typical analysis from the original records, shot records SNR differences, higher SNR 


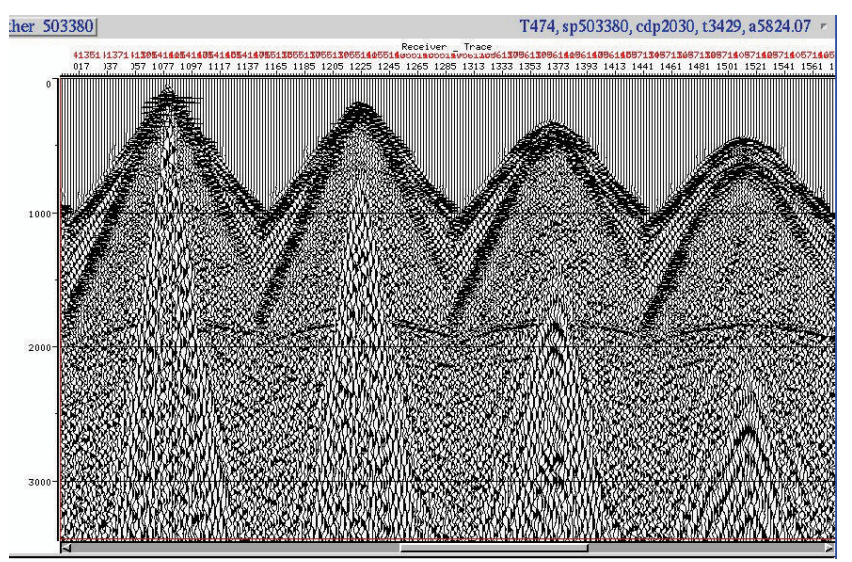

Figure 4: Better quality of the original single shot.

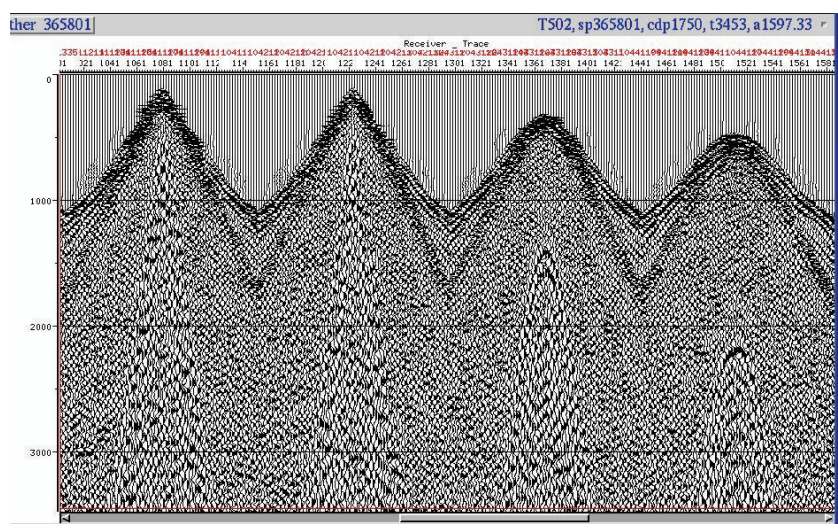

FIGURE 5: Poor quality of the original single shot.

from $1.8 \mathrm{~s}$ to $2.1 \mathrm{~s}$, the whole region can continuously track mark phase axis, with good quality single shot, shallow, midwave group is complete, $2.5 \mathrm{~s}$ or less energy loss is more serious. Poor quality single shot shallow, deep SNR poor record serrated early to beat poor continuity (Figures 4 and 5).

4.1.2. Analysis of the Entire Work Area Stacked Section. Since the surface area of seismic and geological conditions is complex, the entire work area accounts for about $7 \%$ of Salt Lake wetlands; desert area is about $50 \%$; grass and sand grass are about 35\%; rock outcrop is about $8 \%$. Overall, qualities of collected data are quite different; SNR and continuous quality display banded regional variation, and desert zone data was poor, which is shown in Figure 6. This requires us to do information processing, especially denoising before and after stacking, and parameters should be targeted to ensure data consistency.

4.1.3. Interference Wave Analysis. The original single shot shows that work area noise is relatively developed (Figure 7), which can be grouped into several categories:

(1) Mechanical interference: performance on the record is outliers and abnormal trace, and characteristics of such interference is with strong randomness

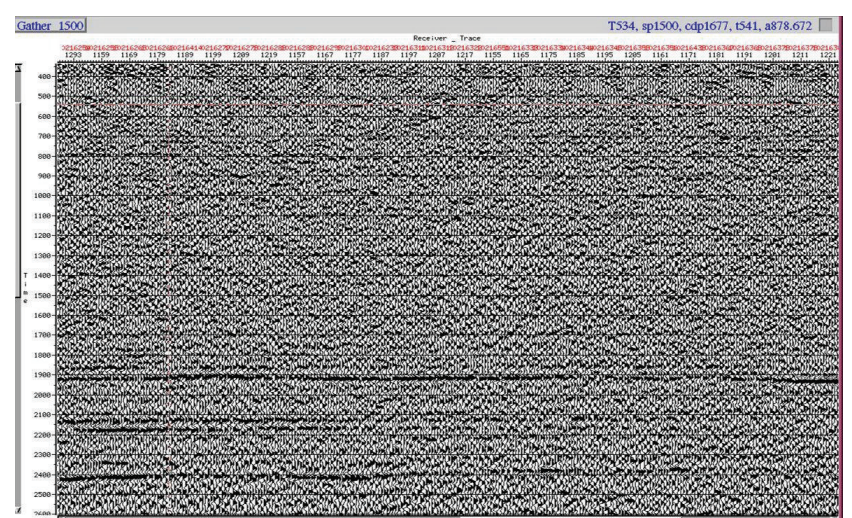

FIGURE 6: Stacked section INLINE551 CDP691-961.

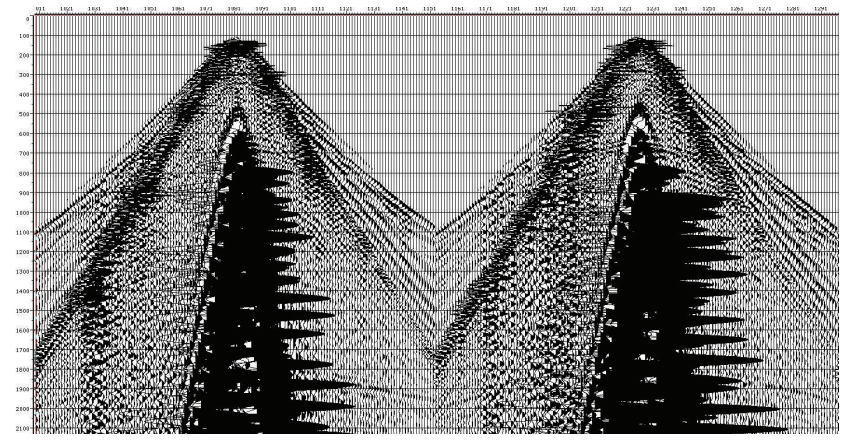

FIGURE 7: Refracted waves, multiple refraction, direct waves, surface waves, and sound waves.

distribution and different stimulation time; different positions have different distributions, easily recognizable from the amplitude [8].

(2) Linear ramp interference: it includes multiple refraction, surface wave, acoustic and some secondary oblique interference, multiple refraction, and secondary oblique interference which are with larger apparent velocity on the record, generally more than $1000 \mathrm{~m} / \mathrm{s}$, with low-frequency surface waves, mainly distributed in the gun point neighborhood, single shot on the record as low apparent velocity, generally not more than $800 \mathrm{~m} / \mathrm{s}$, with high sound waves frequency and strong energy.

(3) Random interference.

Combined with the above analysis of raw data, we can see that interference of three-dimensional low-frequency surface wave is strong in the study area, effective reflections on the original record are full inundated by surface wave, with low SNR; in addition, shallow direct wave interference is strong, with broad band distribution, which seriously interferes with shallow reflection signals [6]. During the processing, we should focus on the basis of noise characteristics analysis, take a targeted approach for noise pressure, carry out noise suppression in a multidomain, and improve SNR of data. Minimizing the effective wave damage in the process of 


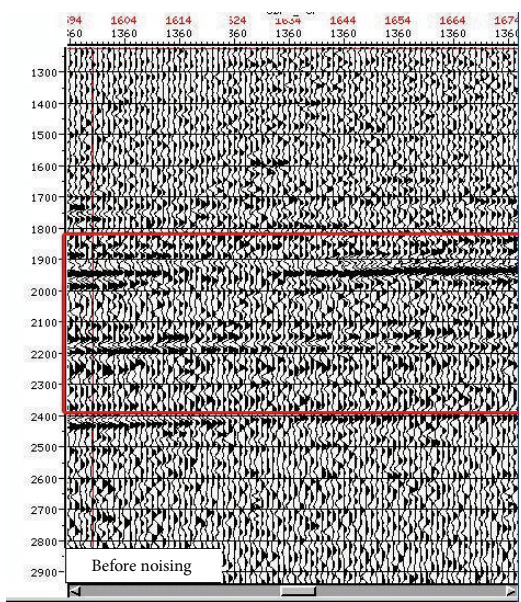

(a)

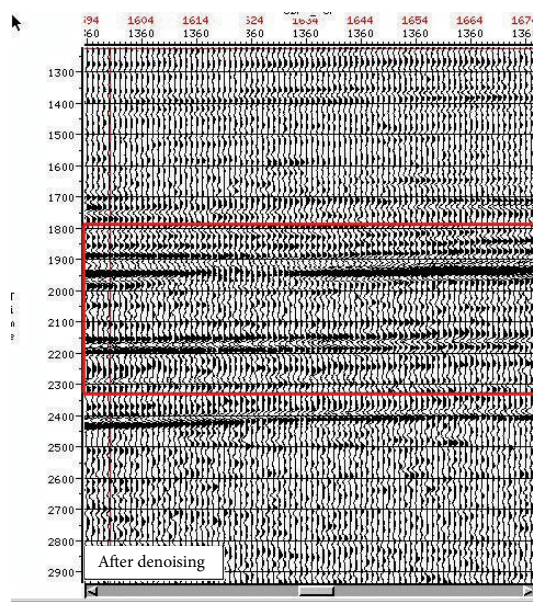

(b)

FIGURE 8: Actual seismic records.

denoising and achieving high-fidelity denoising is another difficulty and emphasis of three-dimensional processing.

4.2. Analysis of the Actual Processing Effect. To illustrate the effectiveness of the proposed denoising method, we selected a small cross-sectional view of seismic data of a work area to actual test. Where Figure 8(a) is actual seismic data with random noise, Figure $8(\mathrm{~b})$ is seismic data denoising with a new threshold method.

The premise of denoising is without prejudice to the effective reflection information, for low SNR data; if it cannot well suppress noise, it is difficult to have effectively phase axis. In order to achieve better denoising effect, after several trials comparing, one finds that using this method is superior to conventional denoising methods [7, 9]. By comparing the two figures, we can see that the noise is significantly reduced after processing the new threshold; meanwhile the resolution has been improved. Thus the new method of denoising seismic data has some practical significance.

From the results, the data for the effective band are from 10 to $70 \mathrm{~Hz}$; frequency ranges are from 13 to $45 \mathrm{~Hz}$, which reached the processing demands and meet the requirements of seismic data interpretation and reservoir inversion. To the whole cross section, the processing result is ideally effective, and SNR of profile and resolution are moderate, with natural wave, and the main target layer wave groups characteristics are distinct, clear, stable, reliable, and with good profile backgrounds, which achieve good results. This confirms the effectiveness of using data denoising algorithm for the complex seismic and geological conditions blocks. (Figures 9 and 10).

To further verify compliance between denoising method and actual seismic exploration, we selected well tie cross section of two representative wells in the area to calibrate and analyze, and we found that synthesis record and main borehole-side well layer are highly consistent. Results show that processing outcome and actual drilling revealing geological features is very accordant (Figures 11 and 12).

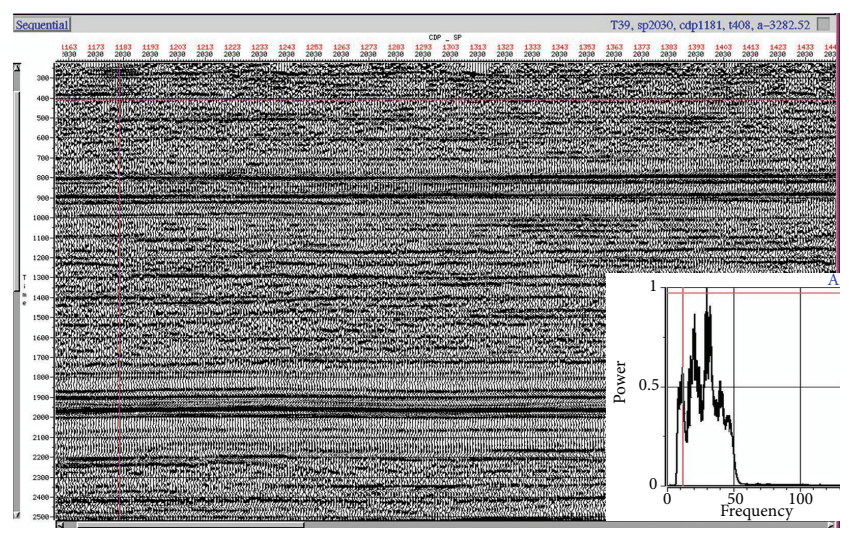

FIGURE 9: Stacked section INLINE1081.

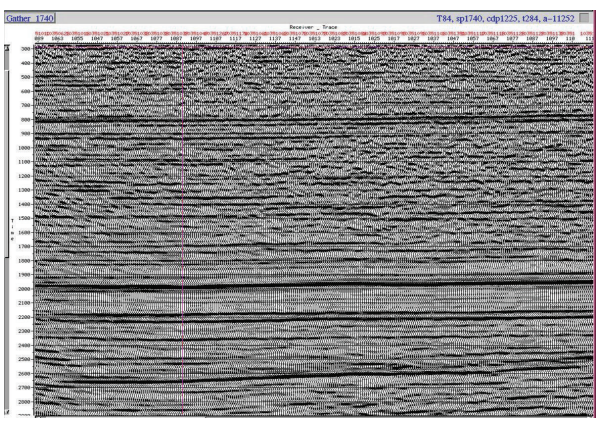

FIGURE 10: Poststack migration profile (INLINE791 CDP194-476).

\section{Conclusions}

Based on wavelet threshold methods proposed by D. L. Dohono and I. M. Johnstone, we present a new threshold function, which enables the superiority of threshold wavelet transform method fully realized. In order to test the effectiveness of the proposed method, we first made ideal synthetic seismograms and achieved the desired results. Finally, we 

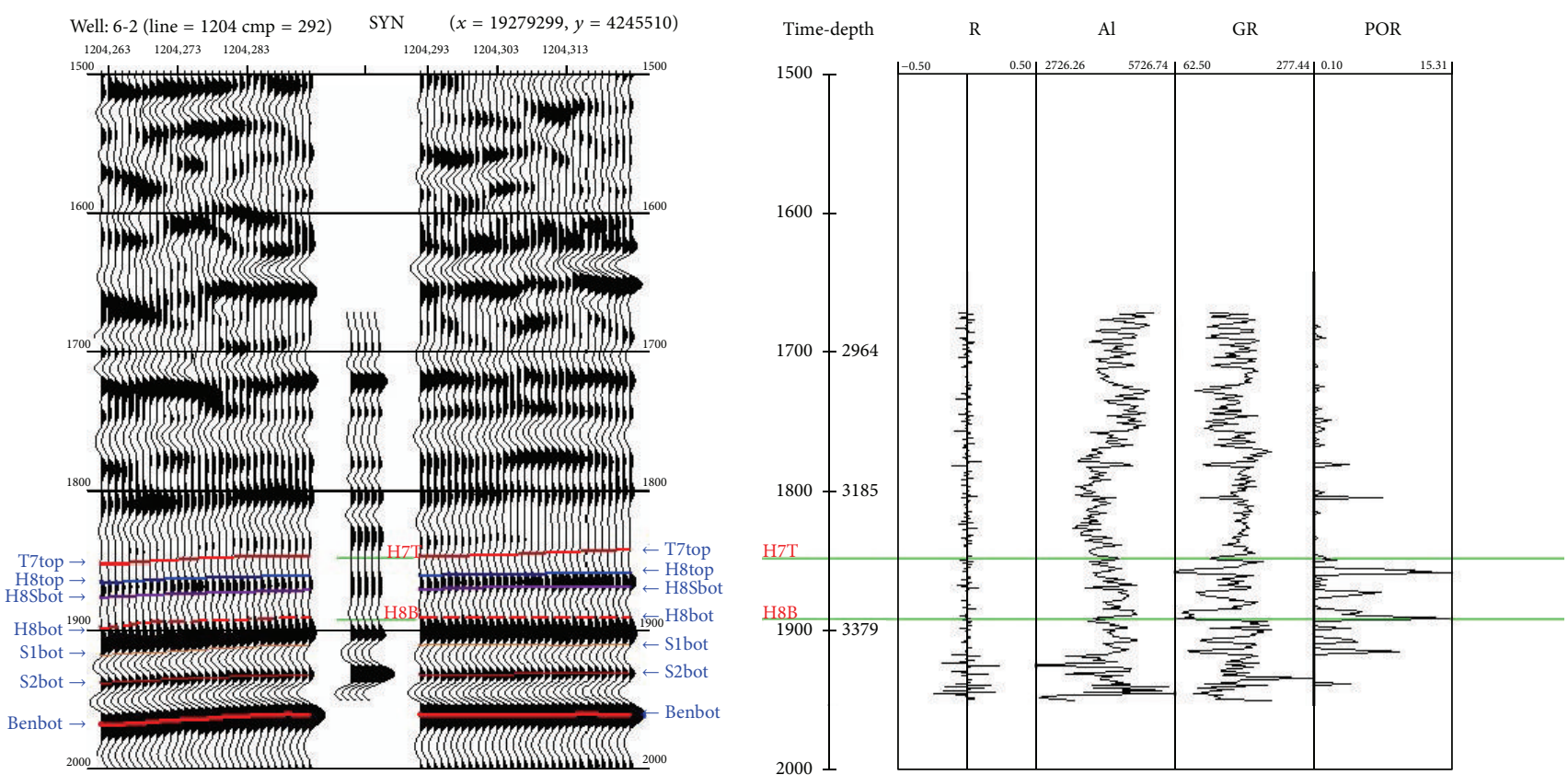

FIGURE 11: X Wells calibration results.
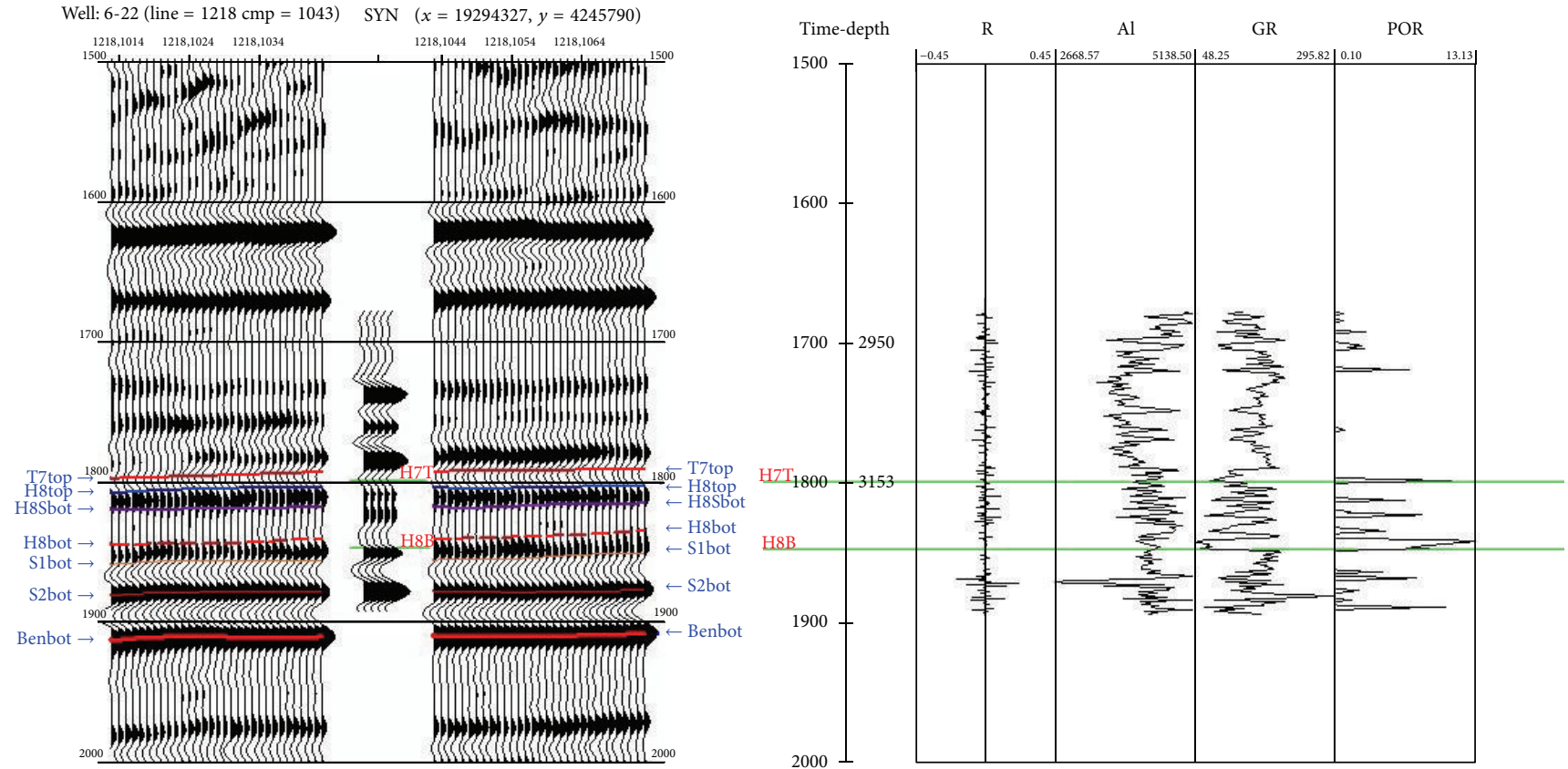

FIGURE 12: Y Wells calibration results.

used the method for denoising actual seismic data, and the results show that this method can effectively remove random noise in original seismic data and improve SNR of seismic data, which provides high quality data for the later work of stratigraphic interpretation and has some practical significance.

\section{Conflict of Interests}

The authors declare that there is no conflict of interests regarding the publication of this paper.

\section{Acknowledgments}

Foundation item: The Joint Specialized Research Fund for the Doctoral Program of Higher Education (20135122110010) and National Natural Science Foundation of China (4127236).

\section{References}

[1] K. Guo and S. Guo, "A method of multiple blind separation based on ICA," Progress in Geophysics, vol. 25, no. 3, pp. 10751080, 2010 (Chinese). 
[2] D. P. Xu and K. Guo, "Reservoir identification generalized S transform and JADE algorithm," Geophysical Prospecting for Petroleum, vol. 4, 2011.

[3] K. Guo, L. Chen, H. Chen, and W. Yang, "The method of seismic random noise blind separation based on JADE," Earth Science Frontiers, no. 3, pp. 302-309, 2011.

[4] S. G. Mallat, "Theory for multiresolution signal decomposition: the wavelet representation," IEEE Transactions on Pattern Analysis and Machine Intelligence, vol. 11, no. 7, pp. 674-693, 1989.

[5] S. Mallat and W. L. Hwang, "Singularity detection and processing with wavelets," IEEE Transactions on Information Theory, vol. 38, no. 2, pp. 617-643, 1992.

[6] T. Liu, X. L. Zeng, and J. Zeng, Getting Practical Wavelet Analysis, National Defense Industry Press, Beijing, China, 2006.

[7] J. Peng, Sensors and Detection Technology, Xidian University Publisher, Xian, China, 2003.

[8] D. L. Donoho, "De-noising by soft-thresholding," IEEE Transactions on Information Theory, vol. 41, no. 3, pp. 613-627, 1995.

[9] Z. X. Cheng, Wavelet Analysis Algorithms and Applications, Xi’an Jiaotong University, Xian, China, 1998. 


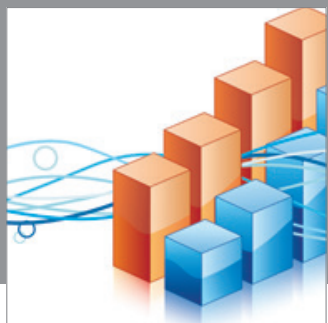

Advances in

Operations Research

mansans

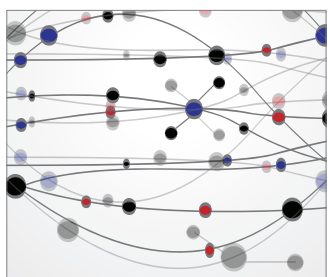

The Scientific World Journal
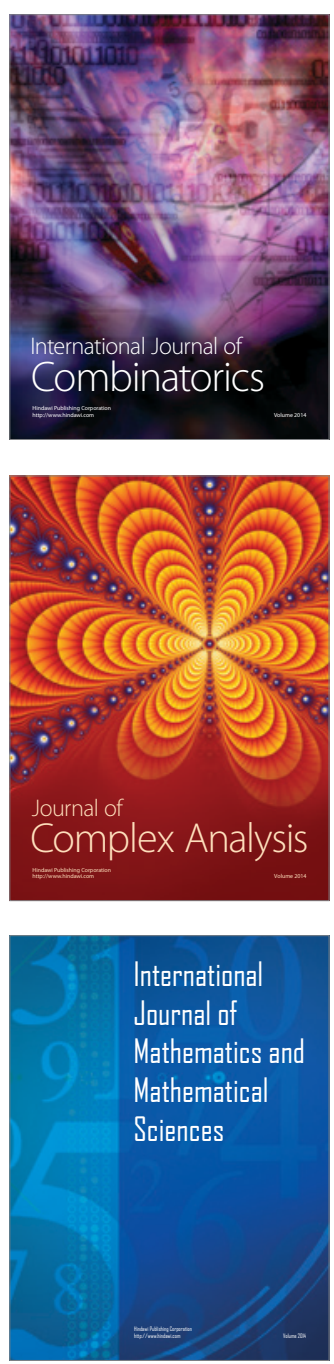
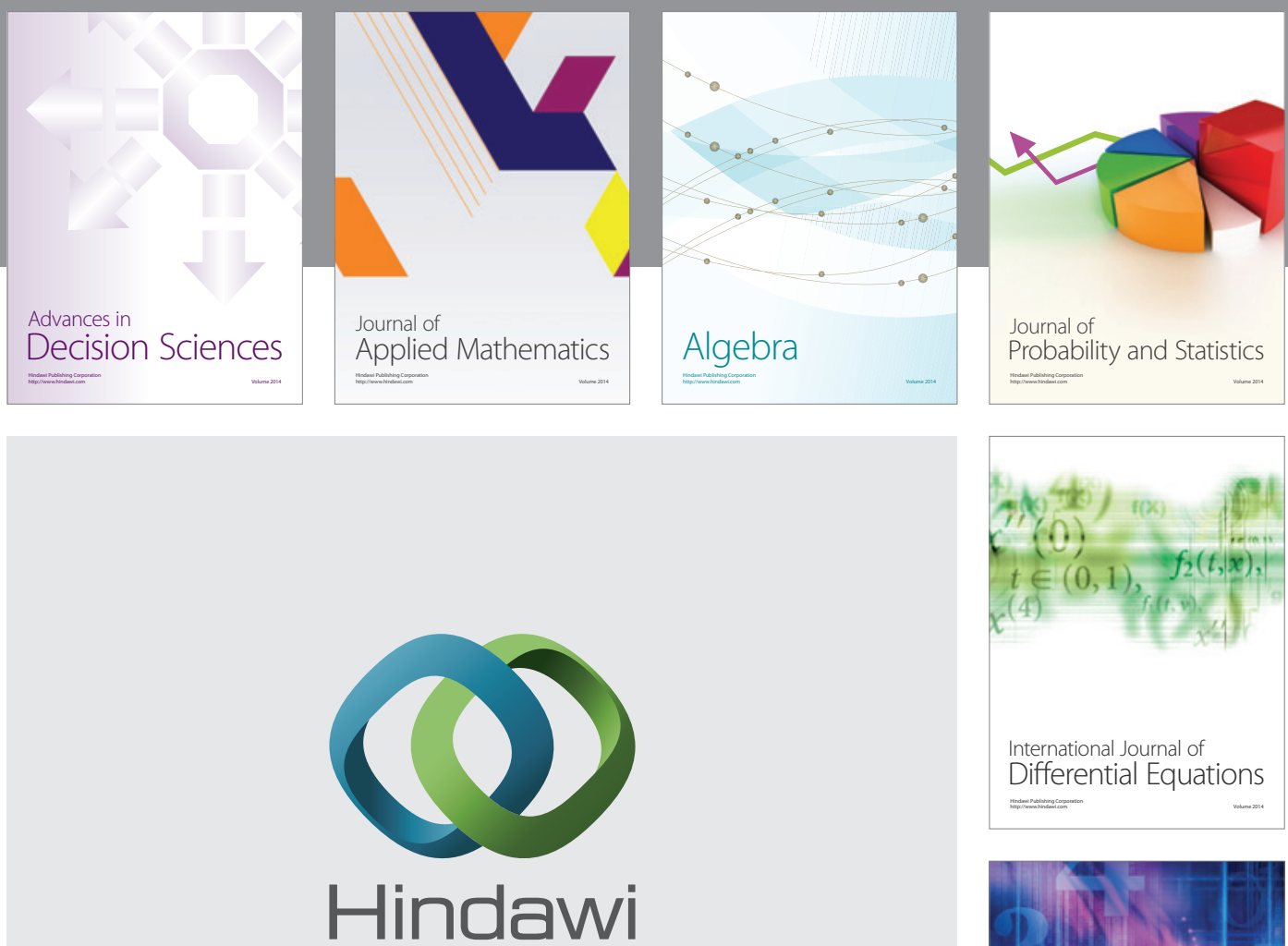

Submit your manuscripts at http://www.hindawi.com
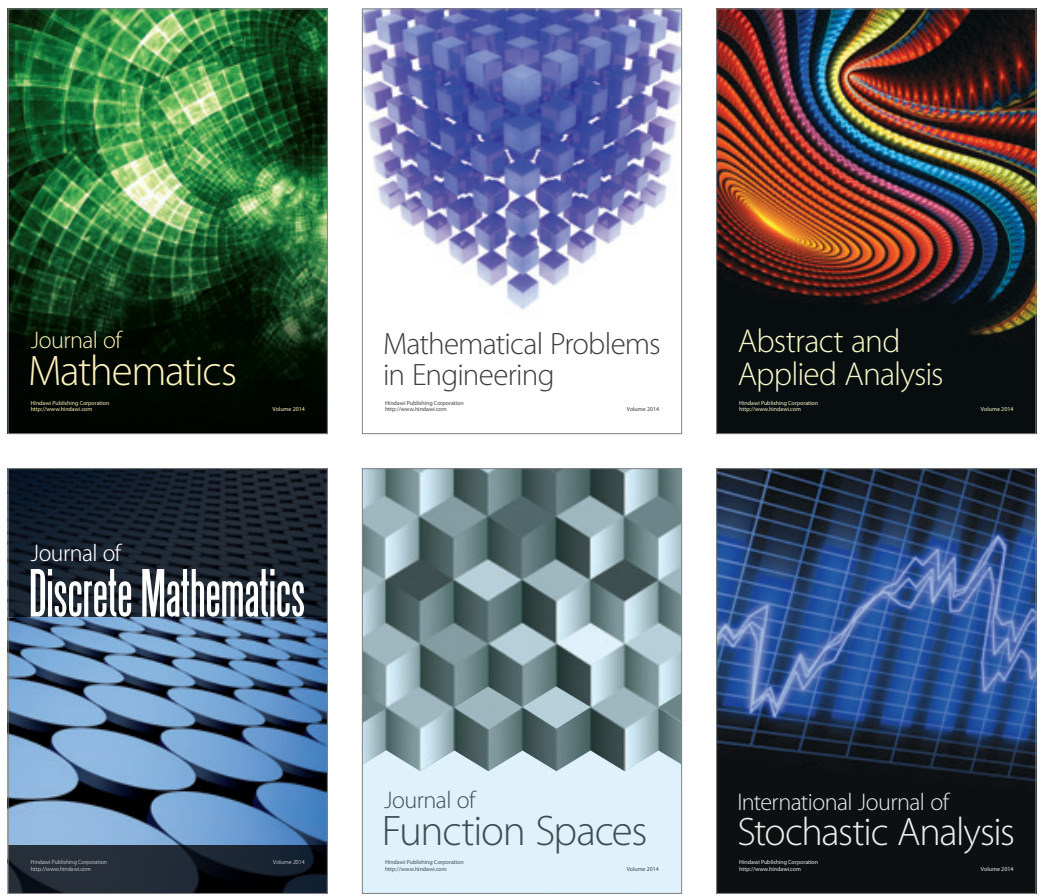

Journal of

Function Spaces

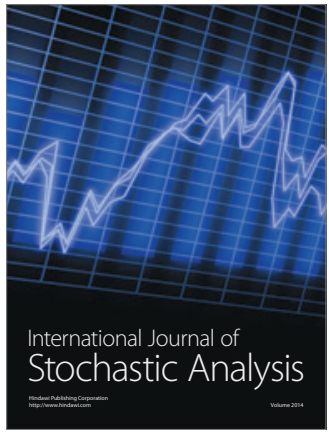

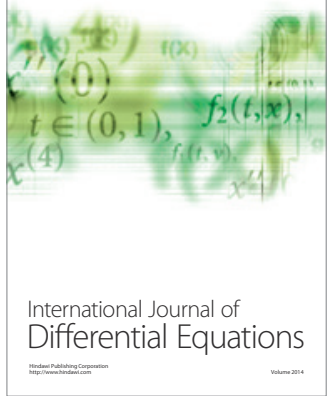
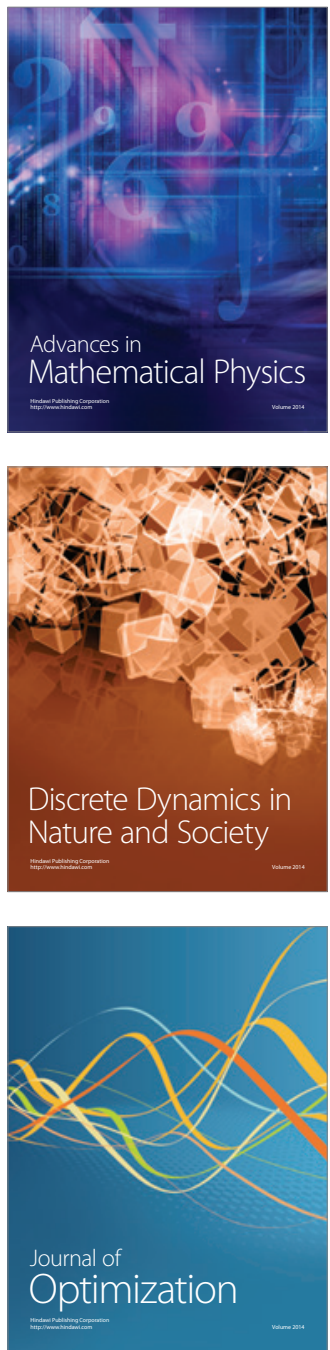